\title{
ON THE ZEROS OF CERTAIN POLYNOMIALS RELATED TO JACOBI AND LAGUERRE POLYNOMIALS*
}

\author{
BY W. LAWTON $\dagger$
}

1. Introduction. We consider the polynomials defined as follows:

$$
\begin{aligned}
J_{n}(x, \alpha, \beta) & \equiv x^{1-\alpha}(1-x)^{1-\beta} \frac{d^{n}}{d x^{n}}\left[x^{n+\alpha-1}(1-x)^{n+\beta-1}\right], \\
L_{n}(x, \alpha) & \equiv x^{1-\alpha} e^{x} \frac{d^{n}}{d x^{n}}\left[e^{-x} x^{n+\alpha-1}\right],
\end{aligned}
$$

where $\alpha$ and $\beta$ are arbitrary real numbers. If $\alpha, \beta>0$, they are known respectively as Jacobi and Laguerre polynomials, satisfying the following orthogonality relations:

$$
\begin{aligned}
& \int_{0}^{1} x^{\alpha-1}(1-x)^{\beta-1} J_{m}(x) J_{n}(x) d x=0, \\
& \int_{0}^{\infty} e^{-x} x^{\alpha-1} L_{n}(x) L_{m}(x) d x=0, \\
& \quad(\alpha, \beta>0 ; m, n=0,1, \cdots ; m \neq n) .
\end{aligned}
$$

From these relations it can be shown that all the zeros of the functions $J_{x}(x, \alpha, \beta)$ and $L_{n}(x, \alpha)$ are real, distinct, and lie respectively inside $(0,1),(0, \infty)$.

The following differential equations are also well known:

$$
\begin{gathered}
x(1-x) J_{n}^{\prime \prime}(x, \alpha, \beta)+\{\alpha-(\alpha+\beta) x\} J_{n}^{\prime}(x, \alpha, \beta) \\
+n(n-1+\alpha+\beta) J_{n}=0, \quad(\alpha, \beta>0), \\
\text { (4) } \quad x L_{n}^{\prime \prime}(x, \alpha)+(\alpha-x) L_{n}^{\prime}(x, \alpha)+n L_{n}(x, \alpha)=0 .
\end{gathered}
$$

Since (3) and (4) represent identical relations between the coefficients of $J_{n}(x, \alpha, \beta)$ and $L_{n}(x, \alpha)$ respectively which are polynomials in $\alpha, \beta$ or in $\alpha$ respectively, we conclude that the differential equations still hold, if $\alpha, \beta \leqq 0$.

* Presented to the Society, March 26, 1932.

$\dagger$ Harrison Fellow in Mathematics, University of Pennsylvania. 
The object of this paper is to study the nature of the zeros of these polynomials when $\alpha, \beta \leqq 0$. In this case the orthogonality relations do not hold since the integrals involved do not exist. Consequently, the aforesaid conclusion about the zeros also fails. M. Fujiwara* has shown that if $p$ and $q$ are positive integers such that

$$
0<\alpha+p<1, \quad 0<\beta+q<1,
$$

then $J_{n}(x, \alpha, \beta)$ has at least $n-p-q$ zeros in $(0,1)$.

In what follows these results have been improved and given in a more precise form (Theorem 2) and similar results derived for $L_{n}(x, \alpha)$ (Theorem 1 ).

2. On the Zeros of $L_{n}(x, \alpha)$ for $\alpha \leqq 0$.

THEOREM 1. (i) If $p$ is a positive integer such that $0<\alpha+p \leqq 1$, $L_{n}(x, \alpha)$ for $n \geqq p$, has exactly $n-p$ zeros inside $(0, \infty)$; (ii) moreover, if $\alpha+p=1, L_{n}(x, \alpha)$ has an additional zero at $x=0$ of multiplicity $p$.

Proof. Case 1. $0<\alpha+p<1$. First, by applying Fujiwara's method, we show that $L_{n}(x, \alpha)$ has at least $n-p$ zeros inside $(0, \infty)$. By (2) we write

$$
\begin{gathered}
x^{\alpha-1} e^{-x} L_{n}(x, \alpha)=\frac{d^{n} \psi}{d x^{n}}, \quad\left(\psi(x)=x^{n+\alpha-1} e^{-x}\right), \\
\int_{0}^{\infty} x^{\alpha+p-1} e^{-x} L_{n}(x, \alpha) x^{m} d x=\int_{0}^{\infty} x^{m+p} \frac{d^{n} \psi}{d x^{n}} d x .
\end{gathered}
$$

(These two integrals exist for $\alpha+p-1>0$.) Furthermore, if $n>m+p$, integration by parts shows at once that the right-hand member vanishes. Hence

(5) $\int_{0}^{\infty} x^{\alpha+p-1} e^{-x} L_{n}(x, \alpha) x^{m} d x=0,(m=0,1, \cdots, n-p-1)$.

Suppose, first, that $L_{n}(x, \alpha)$ has $r(<n-p)$ zeros in $(0, \infty)$ :

$$
\alpha_{1}, \alpha_{2}, \cdots, \alpha_{r}
$$

Then

* M. Fujiwara, On the zeros of Jacobi's polynomials, Japanese Journal of Mathematics, vol. 2 (1925), pp. 1-2. 


$$
L_{n}(x, \alpha)=\left(x-\alpha_{1}\right)\left(x-\alpha_{2}\right) \cdots\left(x-\alpha_{r}\right) P_{n-r}(x) \equiv R(x) P_{n-r}(x),
$$

and (see (5)),

$$
\int_{0}^{\infty} x^{\alpha+p-1} e^{-x} R^{2}(x) P_{n-r}(x) d x=0,
$$

which is impossible, since $P_{n-r}(x)$ does not change sign in $(0, \infty)$. Consequently

$$
r \geqq n-p
$$

Secondly, we show that

Write

$$
r \leqq n-p
$$

$$
L_{n}(x, \alpha)=\sum_{i=0}^{n} \beta_{i} x^{i}
$$

Substituting in (4), we have

$$
(i+1)(\alpha+i) \beta_{i+1}=(i-n) \beta_{i}, \quad(i=0,1, \cdots, n-1) .
$$

Since $0<\alpha+p<1$,

$$
\begin{array}{lll}
\alpha+i<0 & \text { for } & 0 \leqq i \leqq p-1 ; \\
\alpha+i>0 & \text { for } & p \leqq i \leqq n .
\end{array}
$$

Thus, $\beta_{0}, \beta_{1}, \cdots, \beta_{p}$ have like signs, $\beta_{p}, \beta_{p+1}, \cdots, \beta_{n}$ have alternate signs, and the sequence $\left\{\beta_{i}\right\},(i=0,1, \cdots, n)$, present exactly $n-p$ variations in sign. Hence, by Descartes' rule, $L_{n}(x, \alpha)$ has at most $n-p$ zeros in $(0, \infty)$, which, combined with (6), yields the desired conclusion, $r=n-p$.

CAsE 2. $\alpha+p=1$. From (7) we have

$$
\beta_{0}=\beta_{1}=\cdots=\beta_{p-1}=0 ; \quad \beta_{p} \neq 0 .
$$

Thus, $L_{n}(x, \alpha)$ has a zero of multiplicity $p$ at $x=0$.

To show that the remaining zeros lie inside $(0, \infty)$, we write (see (5))

$$
\begin{array}{r}
L_{n}(x, \alpha) \equiv R_{n-p}(x, \alpha) x^{p} ; \int_{0}^{\infty} x^{\alpha+2 p-1} e^{-x} R_{n-p}(x, \alpha) x^{m} d x=0, \\
\quad(m=0,1, \cdots, n-p-1) .
\end{array}
$$

Employing a similar argument to that used in Case 1, we conclude that $R_{n-p}(x, \alpha)$ has at least $n-p$ zeros inside $(0, \infty)$ and therefore exactly $n-p$ such zeros, since it is a polynomial of degree $n-p$. 
3. On the Zeros of $J_{n}(x, \alpha, \beta)$ for $\alpha, \beta \leqq 0$.

THEOREM 2. (i) If $p$ and $q$ are positive integers such that $0<\alpha+p \leqq 1,0<\beta+q \leqq 1$, then $J_{n}(x, \alpha, \beta)$ for $n \leqq p+q+1$ has exactly $n-p-q$ zeros inside $(0,1)$. (ii) If $\alpha+p=1, J_{n}(x, \alpha, \beta)$ has an additional zero of multiplicity $p$ at $x=0$; if $\beta+q=1$, $J_{n}(x, \alpha, \beta)$ has a zero of multiplicity $q$ at $x=1$.

Proof. Case $1.0<\alpha+p<1 ; 0<\beta+q<1$. In view of $\mathrm{M}$. Fujiwara's results, it is sufficient to show that the number of zeros of $J_{n}(x, \alpha, \beta)$ inside $(0,1)$ can not exceed $n-p-q$. This will be done in several steps.

First, we shall show that $J_{n}(x, \alpha+1, \beta)$ has at least one more zero inside $(0,1)$ than $J_{n}(x, \alpha, \beta)$. We get, making use of (1) and of the identity

$$
\begin{gathered}
\frac{d^{n}}{d x^{n}}[\psi x] \equiv x \frac{d^{n} \psi}{d x^{n}}+n \frac{d^{n-1} \psi}{d x^{n-1}} \\
(8) J_{n}(x, \alpha+1, \beta)=J_{n}(x, \alpha, \beta)+n x^{-\alpha}(1-x)^{-\beta+1} \frac{d^{n-1}}{d x^{n-1}} \phi(x), \\
\left(\phi(x)=x^{n+\alpha-1}(1-x)^{n+\beta-1}\right) .
\end{gathered}
$$

Employing the abbreviated notation

$$
J_{n}(x, \alpha+1, \beta)-J_{n}(x, \alpha, \beta) \equiv T_{n}(\alpha)
$$

and differentiating (8), we get, making again use of (1),

(9) $n(1-x) J_{n}(x, \alpha, \beta)=[\alpha-(\alpha+\beta-1) x] T_{n}(\alpha)+x(1-x) T_{n}^{\prime}(\alpha)$.

Differentiating (9) and using (3) written for $J_{n}(x, \alpha, \beta)$ and for $J_{n}(x, \alpha+1, \beta)$, we find

$$
\begin{gathered}
(n-1+\alpha+\beta)\left[J_{n}(x, \alpha+1, \beta)-J_{n}(x, \alpha, \beta)\right] \\
=(x-1) J_{n}^{\prime}(x, \alpha, \beta) .
\end{gathered}
$$

We note that, if $n \geqq p+q+1$, then $n-1+\alpha+\beta>0$.

Let $x_{i}$ and $x_{i+1}\left(>x_{i}\right)$ be two consecutive zeros of $J_{n}(x, \alpha, \beta)$ inside $(0,1)$. Then, comparing the signs of $J_{n}(x, \alpha, \beta)$ and of $J_{n}(x, \alpha+1, \beta)$ in (10) for $x=x_{i}$, and $x_{i+1}$, we conclude that there exists at least one zero of $J_{n}(x, \alpha+1, \beta)$ between $x_{i}$ and $x_{i+1}$.

Next, if $x_{k}$ be the right-most zero of $J_{n}(x, \alpha, \beta)$ inside $(0,1)$, we can show that there exists a zero of $J_{n}(x, \alpha+1, \beta)$ inside 
$\left(x_{k}, 1\right)$. In fact, $J_{n}(1, \alpha, \beta) \neq 0$ (as we shall show later), say $>0$; hence, since

$$
J_{n}^{\prime}\left(x_{k}, \alpha, \beta\right)>0, \quad J_{n}(1, \alpha+1, \beta)>0,
$$

it follows that

$$
J_{n}\left(x_{k}, \alpha+1, \beta\right)<0
$$

by (10).

In a similar fashion, if $x_{1}$ is the left-most zero of $J_{n}(x, \alpha, \beta)$ inside $(0,1)$, there exists a zero of $J_{n}(x, \alpha+1, \beta)$ inside $\left(0, x_{1}\right)$.

Combining the above results, we conclude that $J_{n}(x, \alpha+1, \beta)$ has at least one more zero; hence $J_{n}(x, \alpha+p, \beta)$ has at least $p$ more zeros inside $(0,1)$ than $J_{n}(x, \alpha, \beta)$.

Consider now $J_{n}(x, \beta, \alpha+p)$. The obvious relation

$$
J_{n}(x, \beta, \alpha+p)=(-1)^{n} J_{n}(1-x, \alpha+p, \beta)
$$

shows that $J_{n}(x, \beta, \alpha+p)$ has the same number of zeros inside $(0,1)$ as $J_{n}(x, \alpha+p, \beta)$. We come now to the final step in our proof.

Suppose $J_{n}(x, \alpha, \beta)$ has $n-p-q+k$ zeros inside $(0,1)$, where $k>0$. By the preceding argument $J_{n}(x, \alpha+p, \beta)$ and therefore $J_{n}(x, \beta, \alpha+p)$ have each at least $n-q+k$ zeros inside $(0,1)$. Repeating the argument, we see that $J_{n}(x, \beta+q, \alpha+p)$ has at least $n+k>n$ zeros inside $(0,1)$, which is impossible if $k>0$. Consequently, $k=0$, and our theorem is thus proved for Case 1 .

We can easily prove what was tacitly assumed in the above argument, that $J_{n}(x, \alpha, \beta)$ has no multiple zeros inside $(0,1)$. Suppose $J_{n}(x, \alpha, \beta)$ has a multiple zero at $x_{i}$, so that

$$
J_{n}\left(x_{i}, \alpha, \beta\right)=J_{n}^{\prime}\left(x_{i}, \alpha, \beta\right)=0 ;
$$

from (3)

$$
J_{n}^{\prime \prime}\left(x_{i}, \alpha, \beta\right)=J_{n}^{\prime \prime \prime}\left(x_{i}, \alpha, \beta\right)=\cdots=0 .
$$

Another tacit assumption that $J_{n}(x, \alpha, \beta) \neq 0$ for $x=0,1$ will be revealed in the discussion below.

REMARK. The same results hold, if $0<\alpha+p<1$ and $\beta>0$ (here $q=0$ ) or if $0<\beta+q<1$ and $\alpha>0$ (here $p=0$ ).

CASE 2. $\alpha+p=1,0<p+q<1$. Writing

$$
J_{n}(x, \alpha, \beta)=\sum_{i=0}^{n} \gamma_{i} x^{i}
$$

and substituting in (3), we obtain 


$$
\begin{array}{r}
\{n(n-1+\alpha+\beta)-i(i-1+\alpha+\beta)\} \gamma_{i}+(i+1)(\alpha+i) \gamma_{i+1}=0 \\
(i=0,1, \cdots, n-1)
\end{array}
$$

Hence

$$
\gamma_{0}=\gamma_{1}=\cdots=\gamma_{p-1}=0 ; \quad \gamma_{p} \neq 0
$$

(since $\alpha+p-1=0$ ), which shows that $x=0$ is a zero of multiplicity $p$ of $J_{n}(x, \alpha, \beta)$, so that

$$
J_{n}(x, \alpha, \beta) \equiv x^{p} R_{n-p}(x, \alpha, \beta) .
$$

In the same manner, as we showed for $L_{n}(x, \alpha)$, we can show that $R_{n-p}(x, \alpha, \beta)$ has at least $n-p-q$ zeros inside $(0,1)$. To find an upper limit for the number of these zeros, we substitute in (10)

$$
\begin{aligned}
J_{n}(x, \alpha, \beta) & \equiv R_{n-p}(x, \alpha, \beta) x^{p} \\
J_{n}(x, \alpha+1, \beta) & \equiv R_{n-p+1}(x, \alpha+1, \beta) x^{p-1},
\end{aligned}
$$

and obtain

$$
\begin{gathered}
(n-1+\alpha+\beta) R_{n-p+1}(x, \alpha+1, \beta)=x(x-1) R_{n-p}^{\prime}(x, \alpha, \beta) \\
+[(n+p-1+\alpha+\beta) x-p] R_{n-p}(x, \alpha, \beta)
\end{gathered}
$$

By an argument similar to that given before, (12) shows that $R_{n-p+1}(x, \alpha+1, \beta)$ has at least one more zero inside $(0,1)$ than $R_{n-p}(x, \alpha, \beta)$. Suppose now $R_{n-p}(x, \alpha, \beta)$ has $n-p-q+k$ zeros inside $(0,1)$, where $k>0$. Then $R_{n}(x, \alpha+p, \beta)$ has at least $n-q+k$ zeros inside $(0,1)$. But

$$
R_{n}(x, \alpha+p, \beta) \equiv J_{n}(x, \alpha+p, \beta)
$$

has exactly $n-q$ zeros inside $(0,1)$, as was shown in Case 1 . Thus, $k=0$, and Theorem 2 is established for Case 2 .

CASE 3. $0<\alpha+p<1, \beta+q=1$. From the above argument (see (11)) we can state immediately that $J_{n}(x, \alpha, \beta)$ has a zero at $x=1$ of multiplicity $q$, and exactly $n-p-q$ zeros inside $(0,1)$.

CASE 4. $\alpha+p=\beta+q=1$. It follows from Cases 2 and 3 that $J_{n}(x, \alpha, \beta)$ has zeros at $x=0,1$ of multiplicity $p, q$ respectively. Writing

$$
J_{n}(x, \alpha, \beta) \equiv x^{p}(1-x)^{q} R_{n-p-q}(x, \alpha, \beta)
$$

and applying M. Fujiwara's method to $R_{n-p-q}(x, \alpha, \beta)$, we readily show that $R_{n-p-q}(x, \alpha, \beta)$ has at least $n-p-q$ zeros inside $(0,1)$; hence, being of degree $n-p-q$, it has exactly $n-p-q$ such zeros. 
4. Remarks. (i) The fact that $L_{n}(x, \alpha)$ has exactly $n-p$ zeros inside $(0, \infty)$ also follows by considering it as a limiting case of $J_{n}(x, \alpha, \beta)$. Suppose $\beta>0,0<\alpha+p \leqq 1$, and consider the transformation $x_{1}=\beta x$. We know that the polynomial

$$
\bar{J}_{n}\left(x_{1}, \alpha, \beta\right)=J_{n}\left(x_{1} / \beta, \alpha, \beta\right)
$$

has exactly $h-p$ zeros inside $(0, \beta)$. On the other hand, by (1),

$$
\bar{J}_{n}\left(x_{1}, \alpha, \beta\right)=x_{1}^{-\alpha}\left(1-\frac{x_{1}}{\beta}\right)^{-\beta} \frac{d^{n}}{d x_{1}^{n}}\left[x_{1}^{n+\alpha}\left(1-\frac{x_{1}}{\beta}\right)^{n+\beta}\right],
$$

and since

$$
\frac{d^{i}}{d x^{i}}\left[x^{h+\alpha}\left(1-\frac{x}{\beta}\right)^{k+\beta}\right] \underset{\beta \rightarrow \infty}{\rightarrow} \frac{d^{i}}{d x^{i}}\left[x^{h+\alpha} e^{-x}\right],(i, h, k=0,1, \cdots),
$$

it follows from (2) that

$$
\lim _{\beta \rightarrow \infty} \bar{J}_{n}\left(x_{1}, \alpha, \beta\right)=L_{n}(x, \alpha) .
$$

(ii) From the argument employed in Section 2, we conclude that inside $(0,1)$ the zeros of $J_{n}(x, \alpha+1, \beta)$ separate those of $J_{n}(x, \alpha, \beta)$ and conversely. The same is true of $J_{n}(x, \alpha, \beta)$ and $J_{n}(x, \alpha, \beta+1)$ and of $L_{n}(x, \alpha)$ and $L_{n}(x, \alpha+1)$, inside $(0,1)$, $(0, \infty)$, respectively.

(iii) The results of Section 2 evidently hold for any finite interval $(a, b)$, the polynomials $J_{n}(x, \alpha, \beta)$ being defined as follows:

$J_{n}(x, \alpha, \beta)=(x-a)^{1-\alpha}(b-x)^{1-\beta} \frac{d^{n}}{d x^{n}}\left[(x-a)^{n+\alpha-1}(b-x)^{n+\beta-1}\right]$.

(iv) The aforesaid property of the zeros of the orthogonal Laguerre and Jacobi polynomials $(\alpha, \beta>0)$, that they lie inside $(0, \infty),(0,1)$ respectively, follows at once from Theorems 1 and 2 , if we make there $p=0, q=0$.

The University of Pennsyluania 